\title{
Plastic Surgery Practice during COVID Times
}

\author{
Deepak Bhaskaran ${ }^{1}$ Aswathy Chandran ${ }^{1}$ Sheeja Rajan T.M. ${ }^{1}$ (1) \\ ${ }^{1}$ Department of Plastic and Reconstructive Surgery, Government \\ Medical College, Kozhikode, Kerala, India \\ Address for correspondence Deepak Bhaskaran, MCh, Department of \\ Plastic Surgery, Government Medical College, Kozhikode, 673008, \\ Indian J Plast Surg 2022;55:54-57. \\ Kerala, India (e-mail: deepakb47@gmail.com).
}

\begin{abstract}
Introduction COVID-19 has affected plastic surgeons like never before. We conducted an all-India survey to find how the practice was affected among public/private sector, reconstructive/aesthetic practice, and consultants/residents. We have proposed some solutions to the identified problems, which are supported by previous literature.

Methods A survey framed in Google forms was circulated through WhatsApp and emails in August 2020. Closed and semiopen questions regarding changes in personal and professional lives, coping strategies adopted, and open questions for suggestions in improving practice, academics and measures to tackle the pandemic were included. Responses were collected in an Excel sheet and analysis done using SPSS software.

Results A total of 220 consented responses were obtained. Public hospital practitioners had to bear the COVID-related administrative as well as executive works, especially residents, which led to anxiety, family concern, burnout, and concern about the loss of skills and academics. Patient interaction was also reduced. Aesthetic surgeons bore more financial loss.

Conclusions Plastic surgeons in India faced decrease in caseload, financial loss,

Keywords

- COVID-19 pandemic

- plastic surgery practice

- survey COVID-related duties, workload for residents, reduced academics, family and mental health problems, difficulty with personal protection equipment (PPE) during surgeries, and queries from patients. These can be solved by doing cases within the limits of protocols and safety, pooling public and private sector for COVID duties, rotating residents' groups to reduce workload, using telemedicine for academics and patient consultations, and providing social support groups to surgeons.
\end{abstract}

\section{Introduction}

The outbreak of novel Coronavirus started in Wuhan, China, in December 2019 and was declared as a pandemic on March 11, 2020. The first case in India was on January 30, 2020, and now around 30 million cases and more than 413091 deaths (as of July 17, 2021) have been reported according to the Ministry Of Health and Family Welfare. Due to occupational hazard, nonessential procedures were reduced $^{1}$.The pandemic has affected patient management and resident training.

We assumed that the pandemic must have affected plastic surgeons in India differently, depending where (private or government) and what (reconstructive vs. aesthetic) they practiced and also on their position in their career (consultant vs. residents). To assess the status of plastic surgery in India, a previous survey was done by Shalabh et al, which mostly focused on the impact on teaching and training of residents. ${ }^{2}$ published online

February 16, 2022
DOI https://doi.org/ 10.1055/s-0041-1740079. ISSN 0970-0358.

\footnotetext{
(c) 2022. Association of Plastic Surgeons of India. All rights reserved This is an open access article published by Thieme under the terms of the Creative Commons Attribution-NonDerivative-NonCommercial-License, permitting copying and reproduction so long as the original work is given appropriate credit. Contents may not be used for commercial purposes, or adapted, remixed, transformed or built upon. (https://creativecommons.org/ licenses/by-nc-nd/4.0/)

Thieme Medical and Scientific Publishers Pvt. Ltd., A-12, 2nd Floor, Sector 2, Noida-201301 UP, India
} 
Our study is intended to add to the evidence by assessing and comparing the impact of the pandemic among the various categories of plastic surgeons.

\section{Materials and Methods}

A cross-sectional study was conducted using a survey with Google forms which was circulated via WhatsApp and email. The survey was active from August 13 to September 4, 2020. Plastic surgery consultants and MCh and DNB trainees of India participated. The questionnaire included consent and closed and semiopen questions regarding the changes in personal and professional lives and coping strategies adopted. Open questions for suggestions in improving practice, academics, and measures to tackle the pandemic were included. The responses entered into Microsoft Excel and were analyzed using SPSS version 25.

\section{Results}

A total of 220 responses were collected. Mean age was 44 years.

-Fig. 1 shows statewise distribution of survey participants.

\section{Comparison of Responses among Various Categories}

(i) Public (39.1\%) versus private sector practice (60.9\%).

About 63.6\% aesthetic practice was done in private clinics ( $p$ value $<0.05$ ). Public sector had more reduction in elective cases. But there was not much reduction in emergency cases in public and private hospitals compared with private clinics. Private sector used online classes more (54.5\%) than public sector $(19.8 \%)(p=0.001)$. As much as $81.3 \%$ of private sector were not involved in COVID duties, only $11.4 \%$ underwent quarantine ( $p$-value $<0.05$ ), and $46.3 \%$ enjoyed a lot of family time during the lockdown. About $76.7 \%$ of public sector were involved in COVID duties ( $p$-value $<0.05$ ) and $43 \%$ did quarantine ( $p$-value $<0.05$ ). Nearly $60.5 \%$ of public doctors were afraid of family members getting infected ( $p$-value $<0.05$ ).

About $64 \%$ of the public doctors incurred earning loss of $<25 \%, 54.5 \%$ of private clinics incurred a loss $>75 \%$, and $35.8 \%$ of private hospitals incurred a loss between 50 to $75 \%$ ( $p$-value $<0.05$ ). About $60.2 \%$ of private doctors used telemedicine, while only $39.5 \%$ of public sector used it ( $p$-value $=0.010$ ). Both sectors do not think telemedicine will reduce their workload and improve patient care. Private sector

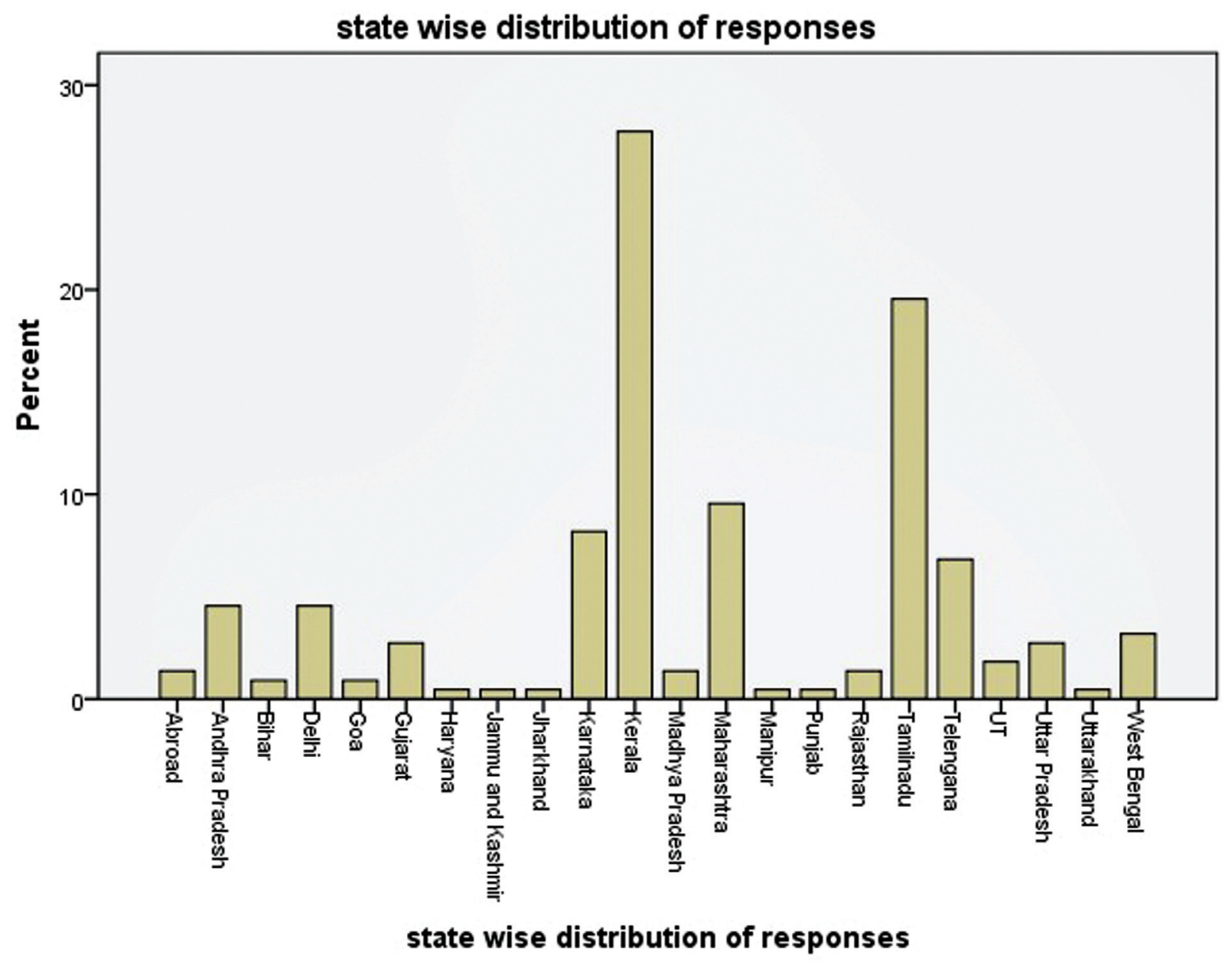

Fig. 1 Percentage of responses-state-wise distribution. 
patients were more concerned about postop care, finance, and contracting COVID. Public sector patients were concerned about the delay in surgery. Nearly $50 \%$ public doctors were concerned about their practical skills, while 54.5\% private doctors were about social interaction with patients. As much as $67.5 \%$ of private surgeons were reluctant to change their old practices even after the pandemic was over $(p$-value $=0.005)$. But public sector doctors were equivocal about this.

\section{(ii) Consultant (83.6\%) versus residents (16.4\%).}

About $75 \%$ residents and $34.2 \%$ consultants performed COVID-related duties ( $p$-value $<0.05$ ). About $71.4 \%$ residents and $20.7 \%$ consultants did quarantine. Some $38 \%$ consultants and $13.9 \%$ residents spent a lot of family time ( $p$-value $<0.05)$. Nearly $52.8 \%$ of residents had spent time with academics during lockdown $(p$-value $=0.006)$. As much as $77.8 \%$ trainees incurred income loss of $<25 \%$, while $85.2 \%$ consultants' incurred losses between was $<25$ to $75 \%$ ( $p$-value $<0.05)$. Some $57.6 \%$ consultants used telemedicine to attend patients, while $75 \%$ residents did not use it. The satisfaction of patient interaction was the major concern for consultants, while residents were worried about the loss of practical skills.

(iii) Aesthetic (11.4\%) versus reconstructive surgeons (88.6\%)

As much as 39.5\% reconstructive surgeons lost about $<25 \%$ of earnings, while $40 \%$ aesthetic surgeons lost between 51 to $75 \%$ ( $p$-value $=0.002$ ). Aesthetic patients were concerned about postop care and traveling to facilities, whereas reconstructive patients were concerned about the delay in surgery and contracting COVID. As much as 63.1\% reconstructive surgeons do not think that telemedicine will reduce their workload, while aesthetic surgeons were equivocal about this. As much as $30.8 \%$ reconstructive surgeon were concerned about loss of practical skills, while it was the satisfaction of patient interaction for $40 \%$ aesthetic surgeons $(p$-value $=0.447)$.

\section{Discussion}

Most of the participants belonged to south and central India with very little input from north and east India. Similar discrepancy was found in a survey done by Shalabh et al in which most participants were from north India. ${ }^{2}$

The reduction in case load in public sector might be due to the fact that public sector hospitals were converted to COVID centers. From our results, public sector was involved in more COVID duties and quarantine than private sector. Public sector was mostly involved in online teaching may be because most public sector hospitals were teaching institutes. Financial loss was mainly for private sector, possibly due to reduction in case load. However, public sector earned their regular salaries from the government. Telemedicine was used mostly in private sector than public sector, which might be due to lack of infrastructure in public sector.
While comparing consultants and residents, residents had to perform more COVID related works and subsequent quarantine. The reason might be that older consultants were not involved directly in COVID duties, considering their age and comorbidities. Subsequently, consultants enjoyed more time with family than residents. However, residents got more time for their theoretical academics during the lockdown. However, they suffered loss of time at acquiring practical skills.

Financial loss of aesthetic surgeons may be due to travel restriction for patients and the decision to reduce nonemergency cases. Majority of plastic surgeons wanted to go back to previous non-COVID practices. Although some needed means to practice their skills, others were concerned about their diminishing income and adverse effects of lockdown on their psyche.

From our survey, the major difficulties faced were decrease in surgical practices, financial loss, COVID-related works, increased workload for residents, reduced academics, family and mental health problems, difficulty with personal protection equipment (PPE) during surgeries, and queries from patients.

We would like to propose some solutions based on the findings from our survey, which are supported by previous literature. Previous literature from Ministry of Health and Family Welfare, articles by Agarwal et al, and Shivangi Saha et al have provided certain guidelines. ${ }^{3-6}$ A study by Rahim et al lists out the framework opted for surgical safety as Kerala model in containing the pandemic. ${ }^{7}$

Problems will be resolved only if our previous practices are restored to some extent, for which recommendations are proposed earlier. ${ }^{6,8-10}$ Suggestions by our survey participants were screening of all patients, using PPE kit, reduction of IP cases and attenders, use of telemedicine to avoid crowded op, asepsis/mask/gloves/social distancing, patient segregation, multiple team approach to reduce infection, and negative pressure theaters.

Türsen et al proposed that aesthetic procedures with lower viral infection risks can be done to prevent emotional stress, anxiety, depression, and isolation of patients during COVID times. ${ }^{10}$ When the strain on health care facilities has reduced and local prevalence and incidence of COVID infection has decreased for at least 2 weeks, elective procedures can be started ${ }^{6,9}$.

We suggest that to reduce COVID-related workload of public doctors, doctors from both public and private sectors can be pooled and duties shared. Hospitals having a shortage of manpower can recruit resource from the pool. Facilities should be made available by the public for interfacility travel and financial providence arranged for private doctors in the pool.

Poore et al suggests reducing residents' workloads by limiting departmental meetings and conducting rounds electronically. Create groups consisting of some faculty and four residents for every 5 days. They complete the rounds, consultations, and emergency cases. A junior resident is kept for surplus cases. Night duties are done by an intermediatelevel resident ${ }^{11}$. 
Loss of academics can be compensated to some extent using online teaching platforms such as Plastiquest, School of PHI, PRS refresher course, and YouTube channels like GK hand surgery or plastic surgery education. Journal clubs, seminars, case discussions, and planning using photos can be conducted online. In a study, students preferred prerecorded classes, as they could attend at any time and at any speed. Most of the students were of opinion that practical classes can never be learned online ${ }^{12}$. Newer resident assessment techniques like online objective structured practical examination (OSPE) and objective structured clinical examination (OSCE) were explored in some centers, considering safety of patients and residents. Communication skills and patient relationship will be compromised. Confidence, discussing plans, and academic presentations are necessary skills. Minimal one-to-one teaching in online platform is a concern. ${ }^{12}$

Health workers have anxiety about personal and family health during pandemics. ${ }^{13}$ Support structures help to find burnout, report symptoms, self-monitor and quarantine, monitor each other for burnout, and schedule "virtual" social time and social time. ${ }^{11}$ According to the Indian Medical Association (IMA), 728 and 730 doctors have succumbed to COVID during the first and second wave, respectively.

Our survey responders said they were unsatisfied with PPE due to poor vision, fogging, difficult to use loupes, headaches, sweating, and heat. Adequate hydration and nutrition should be taken before wearing PPE. Fogging can be reduced by using adequate sealing masks.

According to our survey, telemedicine helped a lot in managing patients. But there was concern about satisfaction of patient interaction. It has been used in trauma and burns and found to be simple, reliable, and cost-effective. ${ }^{14}$ Postoperative follow-up can be conducted using telemedicine. ${ }^{15}$ Nowadays, smartphone applications like "Doctoral diagnosis," help to make a quick diagnosis just by seeing an image. ${ }^{16}$

\section{Conclusions}

The COVID pandemic has affected plastic surgeons differently. Public sector suffered more loss of plastic surgery caseload and were involved more in COVID-related duties. Residents had to do COVID-related duties at the expense of loss of practical skills. However, they got enough time for their theoretical academics during lockdown. Aesthetic surgeons working in private clinics incurred more financial loss compared with others. These difficulties can be solved to some extent by doing cases within the limits of protocols and safety, pooling public and private sector for COVID duties, forming rotatory resident groups to reduce workload, using telemedicine for academics and patient consultations, and providing social support groups to surgeons.
Funding

Nil.

Conflict of Interest

None declared.

\section{References}

1 Shokri T, Saadi RA, Liaw J, et al. Facial plastic and reconstructive surgery during the COVID-19 pandemic: implications in craniomaxillofacial trauma and head and neck reconstruction. Ann Plast Surg 2020;85(2S, Suppl 2)S166-S170

2 Kumar S, More A, Harikar MDharini 1The impact of COVID-19 and lockdown on plastic surgery training and practice in India. Indian J Plast Surg 2020;53(02):273-279

3 MoHFW. Advisory for Hospitals and Medical Education Institutions. 2020;(March):10-12. Accessed September 20, 2021 at: https://www.mohfw.gov.in/

4 Agarwal R, Singhal M, Kant V. Plastic surgery practices amidst global COVID-19 pandemic: Indian consensus. J Plast Reconstr Aesthet Surg 2021;74(01):203-210

5 Saha S, Kumar A, Dash S, Singhal M. Managing burns during COVID-19 outbreak. J Burn Care Res 2020 (e-pub ahead of print). Doi: $10.1093 / \mathrm{jbcr} / \mathrm{iraa} 086$

6 Kaye K, Paprottka F, Escudero R, et al. Elective, non-urgent procedures and aesthetic surgery in the wake of SARS-COVID-19: considerations regarding safety, feasibility and impact on clinical management. Aesthetic Plast Surg 2020;44(03):1014-1042

7 Rahim AA, Chacko TV, Rajan S. Responding to COVID-19: lessons from Kerala on what worked under resource constraint settings and a glimpse into the surgical management of patients. Int J Surg Glob Heal 2020;3(06):e31-e31

8 Brücher BLDM, Nigri G, Tinelli A, et al. COVID-19: Pandemic surgery guidance. 4 Open 2020;3:1

9 American College of Surgeons. Anesthesiologists AS of, Association A of peri Operative RNAH, Introduction. Joint Statement : Roadmap for Resuming Elective Surgery after COVID-19 Pandemic; 2020

10 Türsen Ü, Türsen B, Lotti T. Aesthetic dermatology procedures in coronavirus days. J Cosmet Dermatol 2020;19(08):1822-1825

11 Ethan L, MacKenzie SOP. Slowing the spread and minimizing the impact recommendations for the plastic surgeon. Plast Reconstr Surg 2020;146:681-689

12 Rafi AM, Varghese PR, Kuttichira P. The pedagogical shift during COVID 19 pandemic: online medical education, barriers and perceptions in central Kerala. J Med Educ Curric Dev 2020; 7:2382120520951795

13 Lai J, Ma S, Wang Y, et al. Factors associated with mental health outcomes among health care workers exposed to Coronavirus disease 2019. JAMA Netw Open 2020;3(03):e203976

14 Wallace DL, Jones SM, Milroy C, Pickford MA. Telemedicine for acute plastic surgical trauma and burns. J Plast Reconstr Aesthet Surg 2008;61(01):31-36

15 Ozturk CN, Kuruoglu D, Ozturk C, Rampazzo A, Gurunian Gurunluoglu R. Plastic surgery and the covid-19 pandemic: a review of clinical guidelines. Ann Plast Surg 2020;85(2S, Suppl 2) S155-S160

16 Cervino G, Oteri G. COVID-19 pandemic and telephone triage before attending medical office: problem or opportunity? Medicina (Kaunas) 2020;56(05):250 